galaxies, is thought to have aggregated along threads of dark matter in the early Universe. But the progenitors of today's galaxies are often shrouded in clouds of dust, making it difficult for astronomers to spot them and test this theory.

Hideki Umehata at the European Southern Observatory in Garching, Germany, and his colleagues used the high-resolution Atacama Large Millimeter/ submillimeter Array in Chile to make detailed observations of a narrow slice of the sky. They compared their results with previous surveys of the region to find the galaxies, which were more than 3.4 billion parsecs (11 billion light years) away and producing hundreds of millions of new stars each year.

The study supports the idea that big galaxies form in areas with a high concentration of dark matter.

Astrophys. J. Lett. 815, L8 (2015) OCEAN SCIENCE

\section{Possible pause in Arctic sea-ice loss}

An expected slowdown of large-scale heat circulation in the Atlantic Ocean could temporarily halt the decline of Arctic sea ice (pictured).

Stephen Yeager at the National Center for Atmospheric Research in Boulder, Colorado, and his colleagues used an Earthsystem model to analyse the causes of decadal trends in sea-ice extent in the North Atlantic. They found that the drastic retreat of sea ice since 1990 coincided with a strong Atlantic circulation

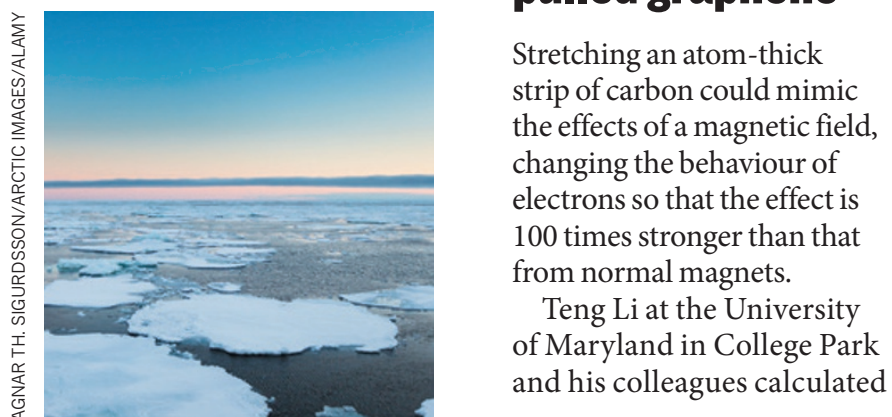

that brought warm surface water from the tropics to high latitudes. If this circulation were to weaken, as observations suggest that it will, less heat arriving in the Arctic Ocean will probably lead to a pause in winter sea-ice loss over the next 5 to 10 years, the authors conclude.

They add, however, that the rate of sea-ice melting could jump back up afterwards as global warming continues. Geophys. Res. Lett. http://doi. org/9wz (2015)

\section{EVOLUTION}

\section{How birds spread around the globe}

The common ancestor of all modern birds lived in South America some 95 million years ago.

Birds inhabit every continent, and are among the most diverse vertebrate groups on Earth. To chart birds' rise and spread, Santiago Claramunt and Joel Cracraft at the American Museum of Natural History in New York created an evolutionary tree based on DNA sequences from 230 bird species and fossil records for 130 extinct species.

They found that bird diversity expanded rapidly after the demise of dinosaurs some 66 million years ago, dispersing along two primary routes. From South America, birds moved into North America, spread to Eurasia through the Arctic and then on to Africa. Birds arrived in Australia by way of Antarctica. Sci. Adv. 1, e1501005 (2015)

\section{Electrons dance in pulled graphene}

Stretching an atom-thick strip of carbon could mimic the effects of a magnetic field, changing the behaviour of electrons so that the effect is 100 times stronger than that rom normal magnets. of Maryland in College Park and his colleagues calculated \title{
SOCIAL SELECTION Romationicas
}

\section{Deleting journal names triggers debate}

Michael Eisen has long argued that research papers should be judged on the basis of their content, not on which journal they were published in. On 6 December, Eisen - a biologist at the University of California, Berkeley, and co-founder of the open-access publisher PLOS - decided to prove his point. He revamped his laboratory's website and announced on Twitter: "Made a new lab website - completely scrubbed any mention of journal titles - http://www.eisenlab.org//publications. html." A few other scientists followed suit, and one even went a step further. Plant geneticist Jeffrey Ross-Ibarra at the University of California, Davis, tweeted: “Following @ mbeisen, removed journal names from website. But also links to cites, almetrics, [sic] \& preprints. http://www.rilab.org/pubs. html." Others were sceptical. Manolis Dermitzakis, a geneticist

S NATURE.COM

For more on

popular papers:

go.nature.com/uwjpkt at the University of Geneva, Switzerland, posted: "I don't see the point. The paper is published in a journal so this is just artificial. Or publish your papers on your website only." how to engineer the large pseudomagnetic fields that are produced when graphene is pulled from two ends. This strains bonds between carbon atoms, causing their electrons to move in a way that is similar to what happens in a magnetic field. The team found that a small tug (of up to $15 \%$ stretch) on certain shapes of graphene strip could produce a strong, nearly uniform field.

The designer shapes could help researchers to study the properties of graphene under extreme conditions - such as large magnetic fields - that are usually unattainable, the authors say.

Phys. Rev. Lett. 115, 245501 (2015)

\section{DEVELOPMENTAL BIOLOGY}

\section{Mini Fallopian tubes in a dish}

Human Fallopian tubes contain adult stem cells that, when grown in the lab, can form miniature 3D structures resembling Fallopian tubes (pictured).

Thomas Meyer at the Max Planck Institute for Infection Biology in Berlin and his

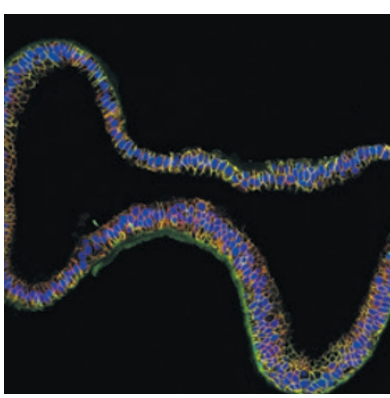

colleagues isolated cells from human Fallopian-tube samples and grew them in $3 \mathrm{D}$ cultures. Two weeks later, they saw mature 'organoids' that had folds in the tissue, hair-like structures called cilia, and secretory cells - all characteristics of the Fallopian tube. The organoids were stable for more than 16 months and sensitive to the hormones oestradiol and progesterone.

The organoids could be used to study tube pathology and certain types of ovarian cancer that are thought to originate in the Fallopian tubes, the authors say. Nature Commun. http://doi. org/9wr (2015)

\section{$\rightarrow$ NATURE.COM}

For the latest research published by Naturevisit:

www.nature.com/latestresearch 